\title{
SNS Accumulator Ring Design and Space Charge Considerations
}

\author{
W. T. Weng \\ AGS Department, Brookhaven National Laboratory, Upton, NY 11973
}

\section{Workshop on Space Charge Physics In High Intensity Hardron Rings}

\author{
May 4-7, 1998
}

Shelter Island, Long Island, NY 


\title{
SNS Accumulator Ring Design and Space Charge Considerations ${ }^{1}$
}

\author{
W.T. Weng \\ AGS Department, Brookhaven National Laboratory, Upton, NY 11973
}

\begin{abstract}
The goal of the proposed Spallation Neutron Source (SNS) is to provide a short pulse proton beam of about $0.5 \mu$ s with average beam power of $1 \mathrm{MW}$. To achieve such purpose, a proton storage ring operated at $60 \mathrm{~Hz}$ with $1 \times 10^{14}$ protons per pulse at $1 \mathrm{GeV}$ is required. The Accumulator Ring (AR) receives $1 \mathrm{msec}$ long $H^{-}$beam bunches of $28 \mathrm{~mA}$ from a $1 \mathrm{GeV}$ linac. Scope and design performance goals of the AR are presented. About 1200 turns of charge exchange injection is needed to accumulate $1 \mathrm{~mA}$ in the ring. After a brief description of the lattice design and machine performance parameters, space charge rela.ted issues, such as: tune shifts, stopband corrections, halo generation and beam collimation etc. will be discussed.
\end{abstract}

\section{INTRODUCTION}

The Oak Ridge National Laboratory is leading a conceptual design for a next generation pulsed spallation neutron source, the Spallation Neutron Source (SNS). There are three major accelerator systems included in Brookhaven's area of responsibility. First is the High Energy Beam Transport (HEBT) system. Secondly, the Accumulator Ring (AR) system and thirdly, the Ring to Target Beam Transport (RTBT) system. This paper describes the design of the AR itself whose magnet and tunnel layout is shown in Fig. 1.

The proton Accumulator Fing is one of the major systems in the design of the SNS. The primary function of the AR is to take the $1 \mathrm{GeV} H^{-}$beam of about $1 \mathrm{msec}$ length from the linac and convert it into a $0.5 \mu$ s beam through a stripping foil in about one thousand turns. The final beam should have $1.0 \times 10^{14}$ protons per pulse, resulting in $1 \mathrm{MW}$ design average beam power at $60 \mathrm{~Hz}$ repetition rate. Provisions have been reserved for a future upgrade to $2 \mathrm{MW}$ beam power by doubling the stored current to $2.0 \times 10^{14}$ proton per pulse without changes in both the magnet and vacuum system [1].

1) Research on the SNS is sponsored by the Division of Material Sciences, U.S. Department of Energy, under contract number DE-AC05-96OR22464 with Lockheed Martin Energy Research Corporation for Oak Ridge National Laboratory. 


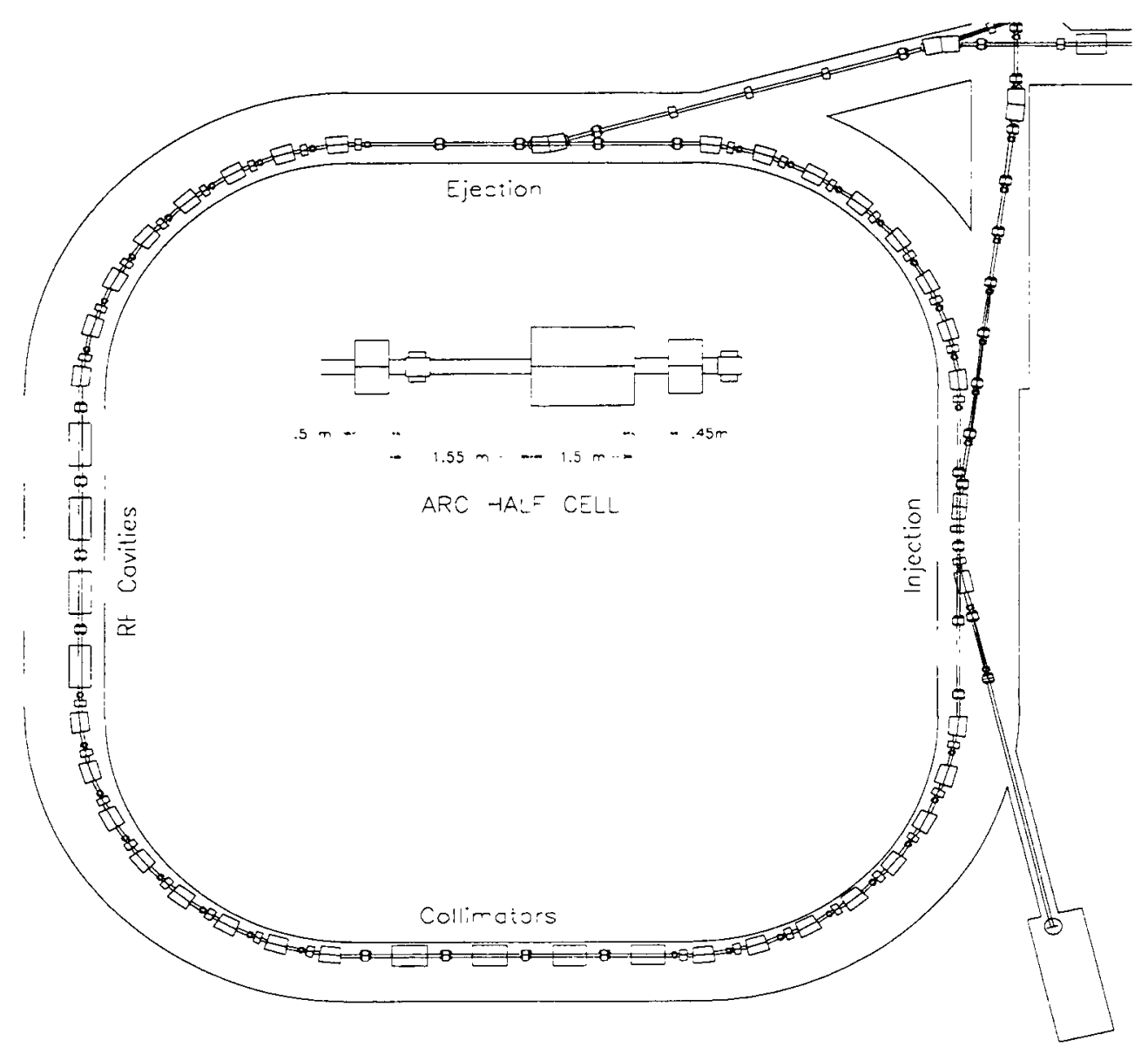

FIGURE 1. Layout of the accumulator ring.

One of the major performance requirements is to keep the average uncontrolled particle loss during the accumulation time to less than $2.0 \times 10^{-4}$ per pulse. The reason of this stringent requirement is to keep the residual radiation to such a level that the hands-on maintenance is possible except for a few localized areas, such as: injection, extraction and collimation. To achieve this goal, special care have been exercised in the $\mathrm{H}^{-}$stripping, the RF stacking, and the collimator design.

In Section 2, the lattice design, $H^{-}$injection and RF stacking process of the SNS accumulator ring will be described. Space charge tune shift and resonance stopband corrections is presented in Section 3. The study in halo formation and its implications on the collimator placement and design is given in Section 4.

\section{LATTICE, $H^{-}$INJECTION AND RF STACKING}

The accumulator ring of the Spallation Neutron Source (SNS) will have a fourfold symmetric lattice. Its lattice function is shown in Fig. 2 [2]. The lattice will accommodate the long straight sections required for the injection system, the 


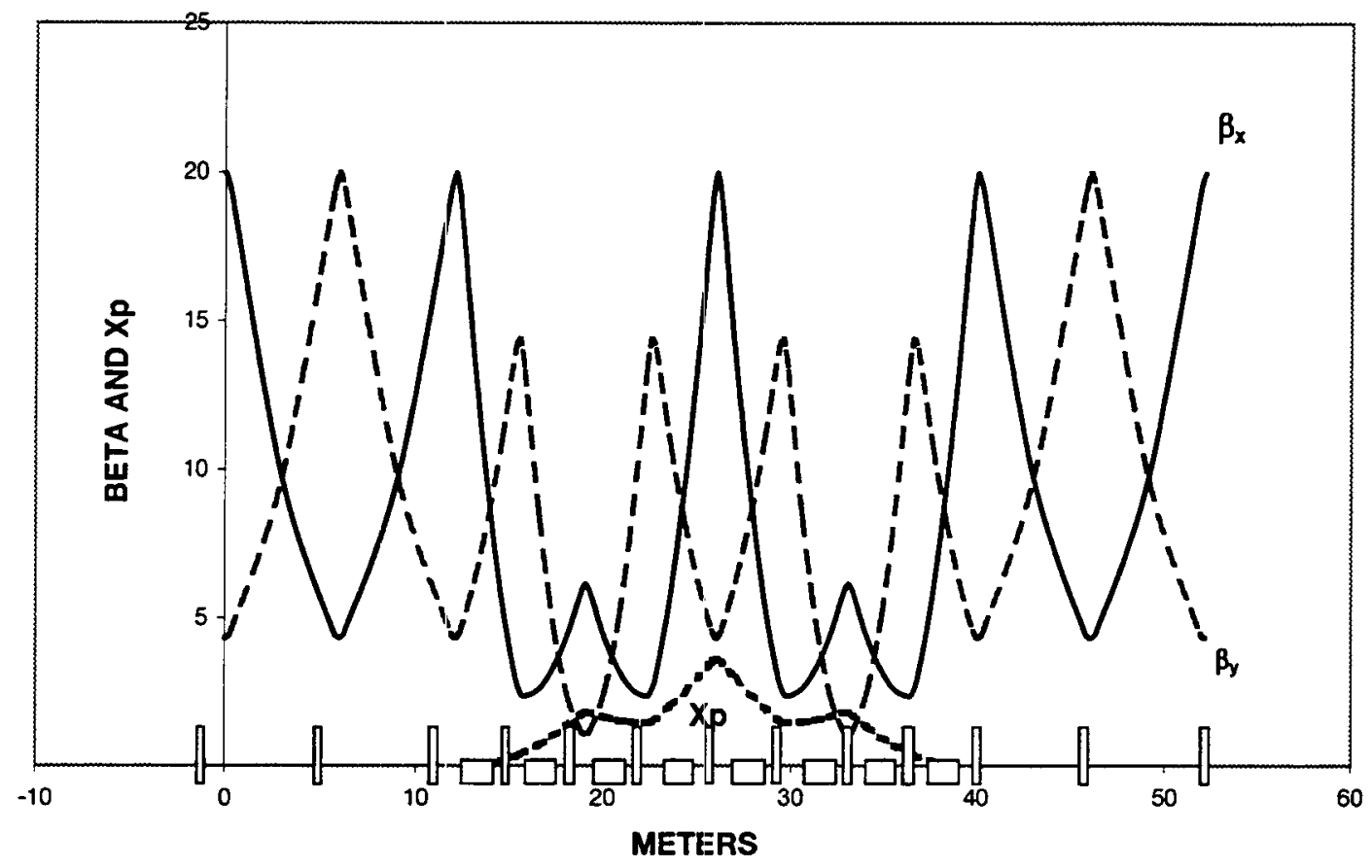

FIGURE 2. Accumulator lattice functions.

extraction system, the RF cavities, and the beam scraping system. The straight sections will be dispersion free, which is desirable, especially for the RF cavities and the injection system. The lattice will provide ease of betatron tuning and flexibility of operation. Unlike lattices of lower symmetry, a lattice of four-fold symmetry will assure that there are no dangerous betatron structure resonances other than for the integer tune. Lattice functions and other salient performance and design parameters of the accumulator ring are summarized in Table 1.

The most demanding system in the design of the $1 \mathrm{MW}$ short pulse spallation neutron source is the $\mathrm{H}^{-}$multi-turn injection into the storage ring [3]. For the SNS accumulator ring, a carbon foil of $400 \mu \mathrm{g} / \mathrm{cm}^{2}$ is assumed. The stripping efficiency for $1 \mathrm{GeV}$ incident $H^{-}$beam is about $99.8 \%$. The temperature rise for the $1 \mathrm{MW}$ design is estimated to be about $3200^{\circ} \mathrm{C}$. In addition, it has been found that

a. Stripping losses in passage of the $H^{-}$beam through the $B=3 \mathrm{kG}$ field in a DC bump dipole magnet vipstream of the stripper foil are negligible.

b. A fraction $f\left(H^{o}\right)=8.19 \times 10^{-3}$ of the incident $H^{-}$beam will emerge as $H^{o}$ from a $400 \mu \mathrm{g} / \mathrm{cm}^{2}$ carbon stripper foil and must be disposed of in an external dump.

c. Field ionization of the $H^{\circ}$ component in the $\mathrm{B}=2.41 \mathrm{kG}$ field of a quadrupole downstream of the foil will lead to negligible uncontrolled losses.

d. Fractional losses from nuclear non-elastic interactions in the foil as low as 
TABLE 1. SNS Accumulator ring parameters

\begin{tabular}{|c|c|}
\hline Beam Average Power & $1.0 \mathrm{MW}$ \\
\hline Kinetic Energy & $1.0 \mathrm{GeV}$ \\
\hline Average Current & $1.0 \mathrm{~mA}$ \\
\hline Repetition Rate & $60 \mathrm{~Hz}$ \\
\hline Ion Source Peak Current & $35 \mathrm{~mA}$ \\
\hline Linac Peak Current & $27.7 \mathrm{~mA}$ \\
\hline Beam Duty Cycle & $6.18 \%$ \\
\hline Linac Pulse Length & $1.03 \mathrm{msec}$ \\
\hline Beam Loss (Controlled/Uncontrolled) & $<4 / 0.02 \%$ \\
\hline Number of Turns Injected & 1158 \\
\hline Revolution Period & $0.8413 \mu \mathrm{sec}$ \\
\hline Revolution Frequency & $1.1886 \mathrm{MHz}$ \\
\hline Circumference & $220.688 \mathrm{~m}$ \\
\hline Space-Charge Tune-Shift & $<0.1$ \\
\hline Bunching Factor (Dual RF Systems) & 0.405 \\
\hline Number of Protons/Ring & $1.04 \times 10^{14}$ \\
\hline Beam Emittance (Transverse, norm.) & $217 \pi \mathrm{mm}-\mathrm{mr}$ \\
\hline Tunes $\nu_{x} / \nu_{y}$ & $5.82 / 5.80$ \\
\hline$\beta_{\max }(\mathrm{x} / \mathrm{y})$ & $19.2 / 19.2 \mathrm{~m}$ \\
\hline Dispersion $X_{p}(\max / \min )$ & $4.1 / 0.0 \mathrm{~m}$ \\
\hline Transition Energy $\gamma_{t}$ & 4.93 \\
\hline RF Voltage ( $1^{s t}$ Harmonic) & $40 \mathrm{kV}$ \\
\hline RF Voltage (2 ${ }^{\text {nd }}$ Harmonic) & $20 \mathrm{kV}$ \\
\hline RF bucket & $17 \mathrm{eV}$-sec \\
\hline Beam Emittance (Longitudinal) & $10 \mathrm{eV}$-sec \\
\hline Beam Gap (injection) & 295 nsec \\
\hline Injected Pulse Length & 546 nsec \\
\hline Extracted Pulse Length & $591 \mathrm{nsec}$ \\
\hline Vacuum & $10^{-9}$ Torr \\
\hline
\end{tabular}

. $1.26 \times 10^{-5}$ can be realized with rapidly (exponentially) collapsing injection bumps and "smoke-ring" injection scheme which result in very small multiple foil traversals of $\left\langle N_{t}\right\rangle=2.43$ traversals/injected proton.

e. Multipole Coulomb and nuclear elastic scattering fractional losses out of a ring acceptance of $A_{x, y}=330 \pi \mathrm{mm}$-mrad for the above injection conditions are $1.35 \times 10^{-5}$, well below our loss criterion of $<10^{-4}$. With the small area stripper foil $(8 \mathrm{~mm} \mathrm{H} \times 4 \mathrm{~mm} \mathrm{~V})$ used for the above estimates, $2.2 \%$ of the incident $H^{-}$ beam misses the foil and will be deflected by a magnet to an external dump.

To accumulate all the particles needed and keep them in proper azimuthal distribution, a dual harmonic RF system is employed. The fundamental RF system will provide $40 \mathrm{kV}$ and the second harmonic RF system will provide $20 \mathrm{kV}$ to form a flattened RF bucket for particle trapping with resulting bunching factor of about 0.4 . Such a RF system will reduce the incoherent tune shift by $25 \%$. The 4 -dimensional multi-particle tracking program Accsim is needed to follow the 1200 turns of par- 
ticles in the ring. The resultant beam distributions in real space and in RF phase space are shown in Fig. 3 and Fig. 4 respectively [4]. Much more work has to be done in this exercise to determine the optimal combination of $R F$ waveform and injection strategy.

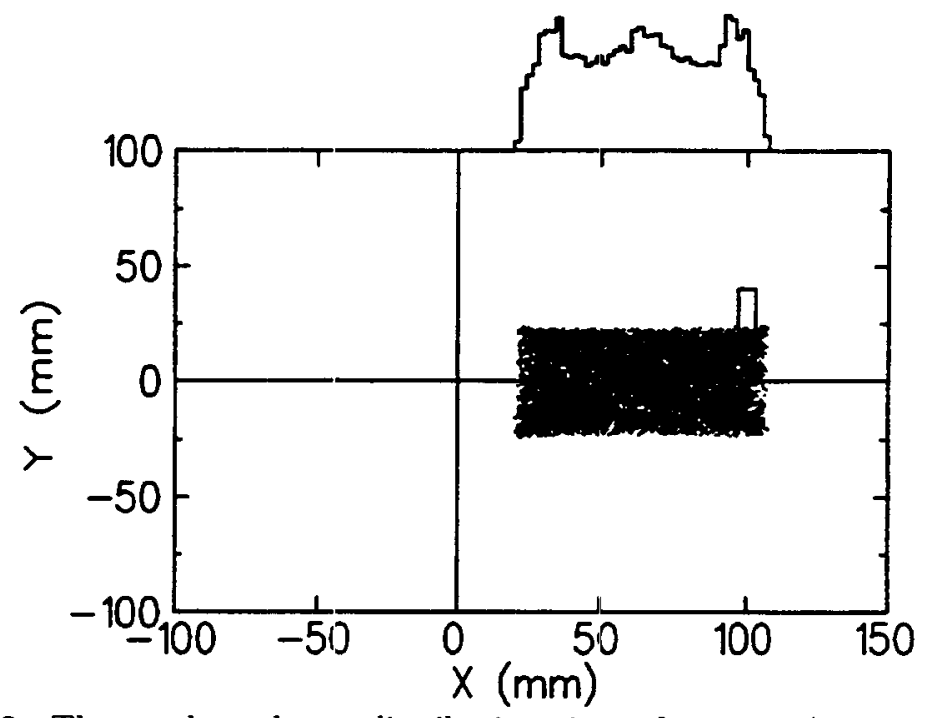

FIGURE 3. The resultant beam distributions in real space. Accsim snapshot at 1200 turns. Pseudo barrier RF.

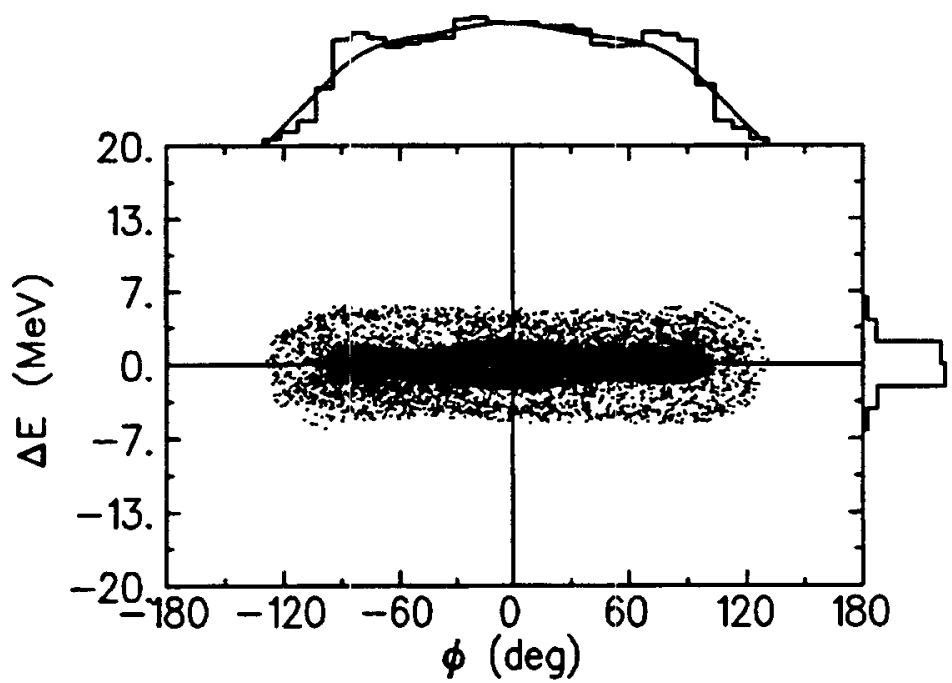

FIGURE 4. The resultant beam distributions in RF phase space. Accsim snapshot at 1200 turns. Pseudo barrier RF. 


\section{SPACE CHARGE TUNE SHIFT AND RESONANCE CORRECTIONS}

\section{A Experiences at the AGS}

We will briefly review the intensity evolution of the AGS from a few $10^{10} \mathrm{ppp}$ to $6.3 \times 10^{13}$ ppp to get some idea of the effect of space charge tune shift at injection.

Shown in Figure 2 is the intensity evolution of the AGS since its completion in $1961[5,6]$. Two major upgrades to the AGS complex were carried out since its operation for physics research in 1961. The first one was the "Conversion Project" from 1967 to 1972 to replace a $50 \mathrm{MeV}$ linac by a $200 \mathrm{MeV}$ linac and to raise the AGS rep-rate from $0.15 \mathrm{~Hz}$ to $0.5 \mathrm{~Hz}$. The second one was the "Booster Project" from 1987 to 1991 to build a Booster to raise the injection energy from $200 \mathrm{MeV}$ to $1.5 \mathrm{GeV}$ and to provide high intensity high mass heavy ions for RHIC. Major improvements in the intensity record are summarized briefly in the following chronology of Table 2.

TABLE 2. Major improvements in the intensity record

\begin{tabular}{|c|c|c|}
\hline Year & Parameters or Improvements & AGS Intensity \\
\hline \hline 1970 & $\begin{array}{c}50 \mathrm{MeV} \text { linac injector } \\
\text { Space charge limited at injection. } \Delta \nu_{y}=0.3\end{array}$ & $3 \times 10^{12}$ \\
\hline 1976 & $\begin{array}{c}200 \mathrm{MeV} \text { linac H+ injector } \\
\text { Resonance stopband correctors; Transverse damper }\end{array}$ & $10^{13}$ \\
\hline 1990 & $\begin{array}{c}200 \mathrm{MeV} \text { linac H- injection } \\
\text { RF feedforward compensation. } \Delta \nu_{y}=0.55\end{array}$ & $1.6 \times 10^{13}$ \\
\hline 1995 & $\begin{array}{c}1.5 \mathrm{GeV} \text { Booster injection; Resonance stopband correctors } \\
\text { Direct RF feedback } \gamma \text {-transition jump } \Delta \nu_{y}=0.35\end{array}$ & $6.3 \times 10^{13}$ \\
\hline
\end{tabular}

It is clear that raising the injection energy is the most effective way to increase the achievable intensity for a space charge limited low energy proton synchrotron. It is also clear that many accelerator physics manipulations have to come into play to keep all those particles inside the synchrotron. The reason that raising injection energy helps to store more particles is due to the reduction of the incoherent space charge tune shift during injection. A good indication of the space charge effect at injection is given by the expression of incoherent space charge tune shift [7]:

$$
\Delta \nu=\frac{N r_{p}}{2 \beta \gamma^{2} \varepsilon_{N} B_{f}}
$$

where $r_{p}=1.54 \times 10^{-18} \mathrm{~m}$ is the classical proton radius, $N$ is the total number of protons, $\varepsilon_{N}$ is the normalized beam emittance, $B_{f}$ is the bunching factor, and $\beta$ and $\gamma$ are the beam velocity and energy. At the tune shift level about 0.35 unit, the acceptable proton intensity changed from $3 \times 10^{12}$, to $10^{13}$ and to $6 \times 10^{13} \mathrm{ppp}$ at three different injection energies respectively. 
As shown in Table 2, the incoherent space charge tune shift at injection can reach as high as 0.55 unit. That means many particles in the beam can move cross half integer and third integer resonance lines. Properly placed quadrupoles and sextupoles are used to correct the stopband width of those resonances to minimize the amplitude growth. Experiences showed that each correction could account for from few percent to tens of percent of reduction of particle losses.

Due to the strong energy dependence of the amount of tune shift caused by the space charge force, raising injection energy can alleviate this limiting effect. This is also the reason that the US Spallation Neutron Source design chooses to have full energy injection at $1 \mathrm{GeV}$.

\section{B Design for the SNS Accumulator Ring}

Now let's look at the space charge tune shift for the SNS accumulator ring. According to eq. (1), with $N=1.0 \times 10^{14} \mathrm{ppp}, \beta=0.875, \gamma=2.066, B_{f}=0.44, \varepsilon_{N}=217$, the expected tune shift is

$$
\Delta \nu_{S . C .} \simeq 0.1
$$

Such a tune shift is relatively small compared to a value of about 0.3 at ISIS and 0.25 at PSR.

Because the ring will operate at very high intensities for which stringent limits on losses will be imposed, the possibility of beam loss due to resonance excitation must be considered. The second, third and fourth-order resonances between tunes of 5 and 6 are shown in Figure 5 [2]. Although the space-charge tune spread of the beam is expected to be small $(\sim 0.1)$, there are a number of resonance lines sufficiently close to the working point to be of concern. Moreover, even though lines such as those excited by normal and skew quadrupoles are far from the working point, they can cause unfavorable distortions of the betatron functions. The fourfold symmetric lattice allows placement of correction elements so that either even or odd harmonics (in azimuth $\theta$ ) can be produced for the correction of the following resonances. Detailed calculations are underway to determine the required magnet strengths.

a. Second-Order Resonances

The $2 \nu_{x}=11$ and $2 \nu_{y}=11$ resonances can be corrected by exciting the trim windings on the quadrupoles with harmonic 11 in azimuth $\theta$. (To produce an odd harmonic, magnets separated by $\theta=180^{\circ}$ are excited with equal but opposite currents.) Similarly, the sumı resonance $\nu_{x}+\nu_{y}=11$ can be corrected by exciting the skew quadrupole correctors with harmonic 11 ; the difference resonance $\nu_{x}-\nu_{y}=0$ can be corrected (or enhanced if coupling between the two planes is desired) by exciting these correctors with harmonic 0 .

b. Third-Order Resonances

The $3 \nu_{x}=17$ and $\nu_{x}+2 \nu_{y}=17$ resonances can be corrected by exciting sextupoles with harmonic 17 . The eight sextupole correctors allow for independent 


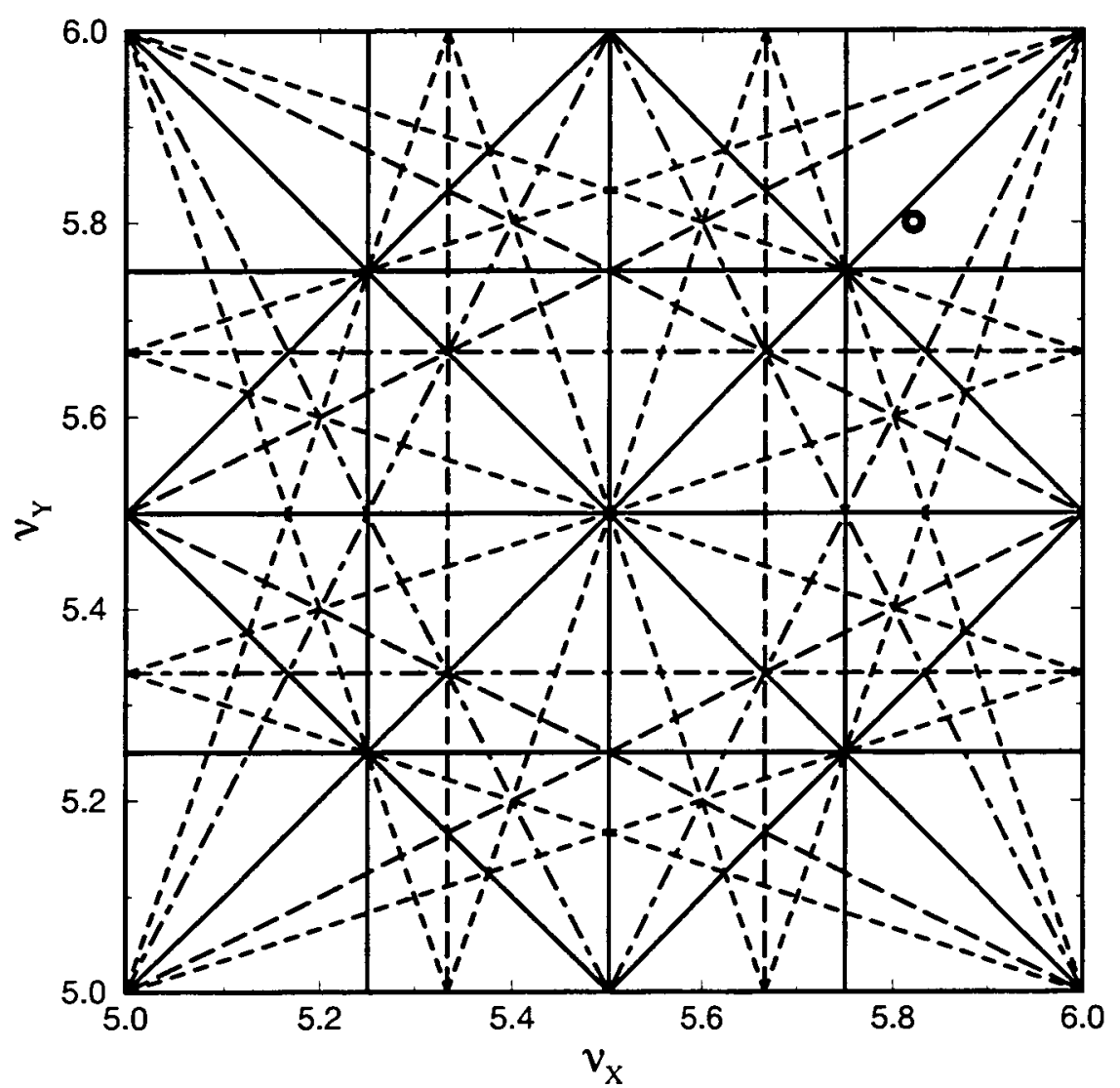

FIGURE 5. Accumulator Tune Chart. The circle shows the working point. Solid and short-dashed lines indicate second and fourth-order resonances; long-dashed and dot-dashed lines indicate third-order.

correction of the two lines. Similarly, the $3 \nu_{y}=17$ and $\nu_{y}+2 \nu_{x}=17$ resonances can be corrected with the eight skew sextupole correctors.

\section{c. Fourth-Order Resonances}

The fourth-order resonance lines are driven by octupoles (both normal and skew) and those closest to the working point pass through the point $\nu_{x}=\nu_{y}=5.75$ in Figure 5 . The effects of these lines can be reduced by exciting the octupole correctors with harmonic 0 . Schemes for correcting specific lines are under investigation. The octupole correctors also allow for Landau damping of transverse instabilities.

\section{d. Structure Resonances}

One consideration in our choice of a lattice was the avoidance of structure resonances. The 4-fold symmetric lattice has fourth-order structure resonances at betatron tunes of 5 and 6; third-order at tunes of 5.333 and 6.666; and secondorder at a tune of 6 . However, none of these are near the proposed working point. A 3-fold symmetric lattice that has been considered in previous design studies, on the other hand, has fourth-order structure resonances at a betatron tune of 3.75 which is right on top of the proposed working point for this lattice. Although these 
resonances may not hurt the ring performance, they are best avoided.

\section{HALO FORMATION AND COLLIMATION}

One of the major performance requirements of the SNS ring is to keep the average uncontrolled particle loss cluring the accumulation time to less than $2.0 \times 10^{-4}$ per pulse. The reason of this siringent requirement is to keep the residual radiation to such a level that the hands-on maintenance is possible except for a few localized areas, such as: injection, extraction and collimation.

Typical beam losses of existing low power proton synchrotron are in the order of a few percent. Let us use the AGS and the proposed SNS ring as example. The relevant beam parameters are summarized in Table 3. It can be clearly seen that $1 \%$ loss of the SNS ring is equivalent to the entire flux of the AGS beam. Such a situation is totally unacceptable.

TABLE 3. AGS and SNS parameters

\begin{tabular}{|c|c|c|}
\hline & AGS & SNS \\
\hline Proton Intensity & $6 \times 10^{13} \mathrm{ppp}$ & $10^{14} \mathrm{ppp}$ \\
\hline Rt:p-Rate & 0.5 & 60 \\
\hline Flux & $3 \times 10^{13} \mathrm{pps}$ & $6 \times 10^{15} \mathrm{pps}$ \\
\hline Los s of $1 \%$ & $3 \times 10^{11} \mathrm{pps}$ & $6 \times 10^{13}$ \\
\hline
\end{tabular}

All existing low energy high intensity proton synchrotrons have operated at a condition that the available physical apertures are fully occupied. Quantitatively, it can be characterized as

$$
\varepsilon_{A} / \varepsilon_{B} \simeq 1.0
$$

where $\varepsilon_{A}$ signifies the equivalent emittance of the aperture and $\varepsilon_{B}$ is the beam emittance. For the SNS accumulator ring design,

$$
\left(\varepsilon_{A} / \varepsilon_{B}\right)_{S N S} \geq 3.0
$$

In other words, there is about factor of 1.6 of space allowed in both vertical and horizontal dimension. The question now is that "is it enough?"

It has been found that the large amplitude particle can interact with the core particles to move either closer to the center or away from the center [8-10]. This process can be understood by an envelope oscillation created by the mismatch between the beam shape and the lattice of the focusing channels. A particle in the halo region tends to be driven away in such a mismatched focusing channel. Although the smaller amplitude particles stay close to the stable fixed point in the center, the larger amplitude particles can drift away following the multiple islands as show in Fig. 6 [9]. The crucial questions now, are how far the islands can extend away from the center, what are the dynamical nature of the islands, and when the 
chaotic motion will set in. Those are all important questions to be answered by any new high power accelerators. It can happen both in the linac and in the circular rings.

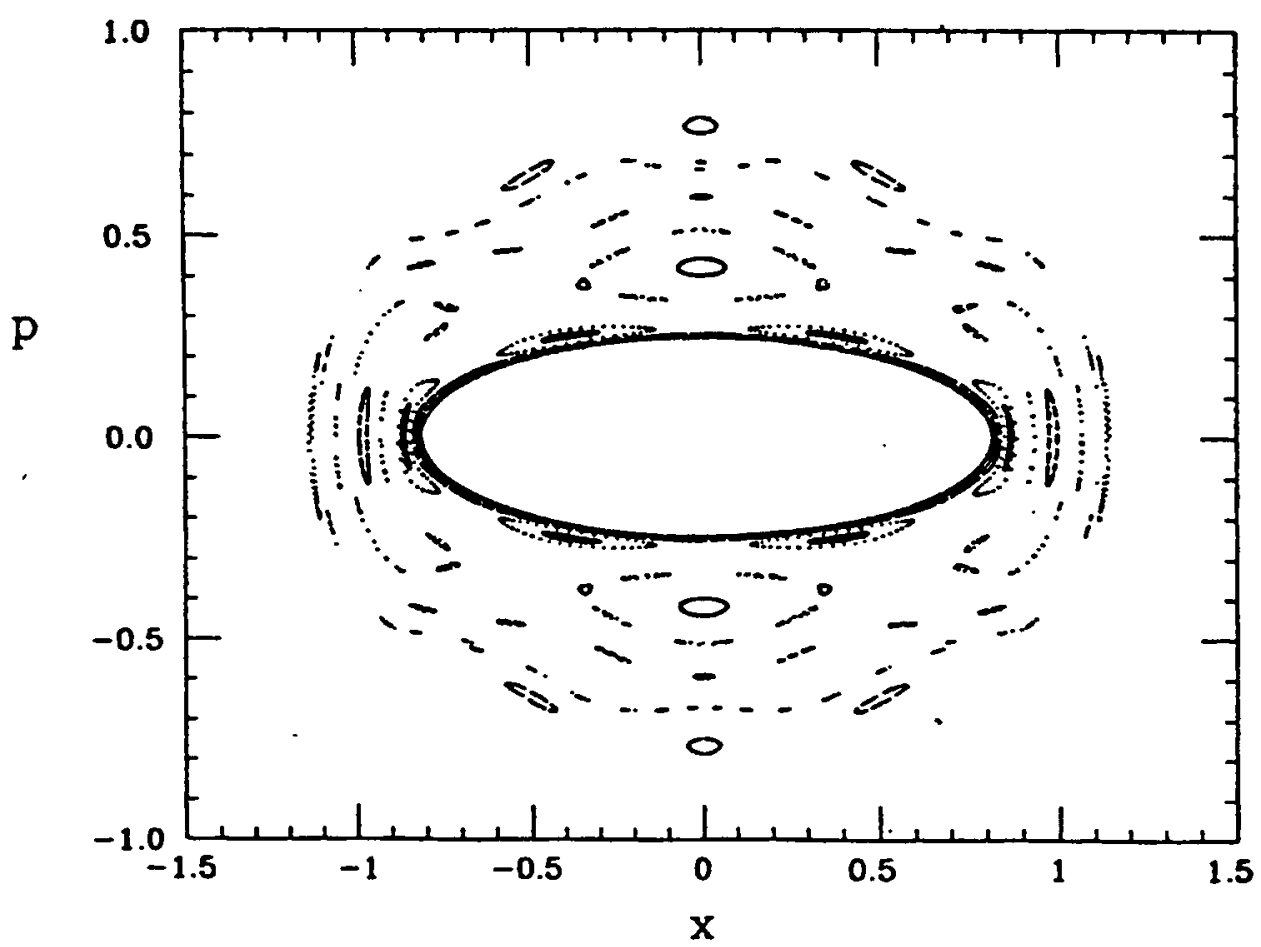

FIGURE 6. The Poincare surface of section in particle phase space from particle-core interaction [9].

A thorough understanding of the halo dynamics as function of mismatch, power supply ripple, space charge tune shift, and the lattice structure, etc., is necessary to be able to estimate the degree of beam losses and placement of collimators with confidence.

Assuming Gaussian distributions shown in Fig. 7, the development of proton synchrotron and main attention of accelerator physics in the past 40 years can be roughly classified into three period.

a. Period 1 located roughly between 1960 to 1975 when the total intensity for fixed target research was a major concern. The figure of merit in this period is the total intensity,

$$
N=\int_{-2.5 \sigma}^{2.5 \sigma} f(z) d z .
$$

b. Period 2 located roughly between 1970 to 1990 when the brightness for colliding beam research was a major concern. The corresponding figure of merit is the brightness, $B=N / \sigma$. 


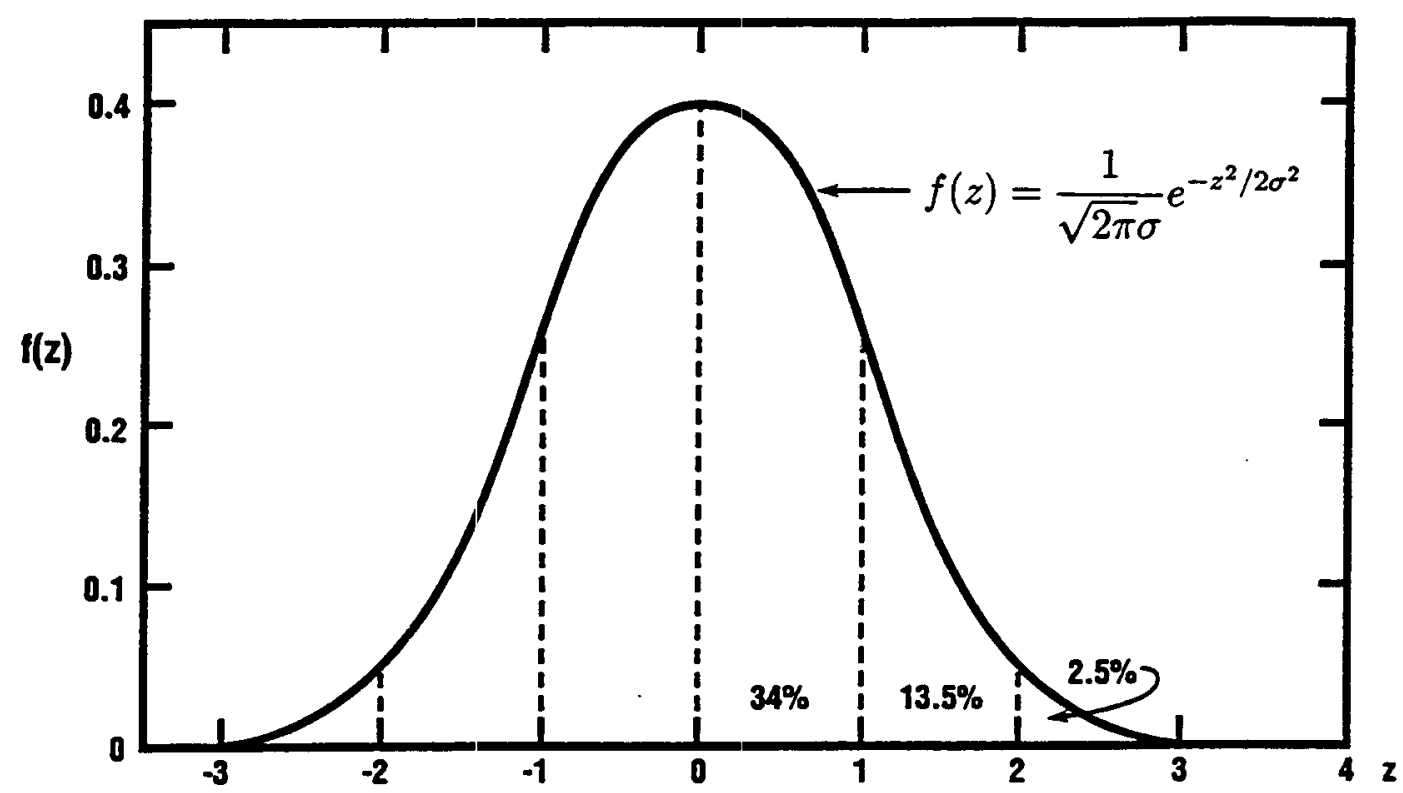

FIGU1RE 7. Typical Gaussian distribution.

c. Period 3 started from 1990 and could well extend to 2010 when the loss of the halo particles is a major concern. This concerns all high power proton accelerators from $1 \mathrm{MW}$ to $10 \mathrm{MW}$ range. The corresponding figure of merit will be the population and dynamics of the halo particles,

$$
H=2 \int_{4 \sigma}^{\infty} f(z) d y .
$$

We heard from $\mathrm{T}$. Wangler that for a tune-depressed and mismatched linac beam, the amplitude growth can reach 5 to 6 times of the beam root-mean-square size. In a synchrotron, both the tune depression and mismatch is less than that of a linac, the maximum amplitude growth should be lower. However, the actual performance can only be understood after: careful tracking studies.

The SNS collaboration initiated two programs to meet the need of reliable and flexible 6-dimensional tracking code for injection and halo studies. Such a code should include space charge effect in both transverse and longitudinal planes following the injection process of turn-by-turn build up from the linac with an aim to push the reliability limit far beyond what is available today for the design of the injection process and the collimation strategy of the SNS accumulator ring. To meet the design requirements, the code should be able to give reliable answer to particle dynamics at large amplitude to better than $1 \%$.

Our first effort is to generate a new code which is called "Sensible Analysis Model for Beams in Accelerators" (SAMBA). Although there are similar codes, such as ACCSIM of TRIUMF, Simpson of KEK and Track2 of ESS, we feel that the SNS project needs a specialized code, that could be developed in parallel by all the 
members of the joint team, and is more comprehensive than existing codes, yet devoid of things that are not so important for our project. In doing so, we have freely borrowed from other codes we know, trying to collect the best in each one of them. We report the development of simulation codes by the joint team. The salient feature of this new code is summarized in the following [11].

SAMBA is written in $\mathrm{C}++$. We did this for two main reasons:

- development of programming modules in parallel is more natural with $\mathrm{C}++$ than with Fortran. This is why $\mathrm{C}++$ has become the standard in the Industry, where a large group of programmers are often working on the same code, to bring it as early as possible to the market.

- the code is run under the supervision of a SuperCode that allows the coexistence of compiled and interpreted modules, making development and debbuging very natural, as it will be explained later in detail.

the machine optics code that produces the main accelerator descriptor is MAD. This is a well developed and maintained code able to describe all of the features of the ring lattice, including higher order transformation matrices and the treatment of lattice errors.

SAMBA is independent from any specific graphic packages. Output to graphics can be done through files or by direct pipe from the code to graphics, in order to allow for animation. An intriguing possibility is to use the graphic firmware tools of the workstation to perform some of the calculations.

The real beam is represented by an ensemble of macro-particles in fully 6dimensional phase space. The calculations include:

a. transport of the particles in the lattice using the first order and second order transformations calculated by MAD.

b. in the transverse space, space charge forces are calculated that apply an angle kick to the macros. Betatron tune distribution in the beam is calculated ("necktie"). Transverse impedances are used to calculate beam to wall coupling in a realistic vacuum chamber geometry.

c. in the longitudinal space, we have cavities with $R F$ voltage plus a realistic longitudinal impedance budget, that allows the representation of longitudinal space charge kicks and the description of the bunch and the bucket.

Most of our design work on SNS injection and RF stacking has been performed using SAMBA. Work is continuing to complete this code development.

Since the most crucial physics input in this tracking code is the treatment of space charge force, to save time, some kind of approximations are always made. Such a simplified approach is necessary in comparing one scenario from another. However, its accuracy has to be checked by more reliable approach. To establish a benchmark for simplified treatment and establish accuracy of the calculation we create a particle in cell (PIC) module for space charge calculation. 
Previous injection calculations for the SNS have employed the Schonauer model [12] for transverse space charge effects, which are limited to shifting the the macro-particle tunes. No other space charge effects on the transverse spatial charge distributions are calculated in this model. As such, to date the final ring emittance is determined by the choice of the linac beam distribution, by the closed orbit bumps, and by scattering of the beam by the foil. In this model only foil interactions produce beam halo. Here, preliminary results are reported using a full Particle-In-Cell (PIC), space charge calculation [13] for SNS injection. The impact of transverse space charge on emittance growth and halo are examined by repeating the previous Schonauer model optimized injection scenarios, here using the PIC model. Although the initial operation of the SNS will be at 1.0MW of beam power, these calculations are done for $2.0 \mathrm{MW}$ of beam power, since the ring is designed to allow for the $2.0 \mathrm{MW}$ upgrade.

We have two specific results to show following the space charge treatment of particle-core model using PIC. Due to the preliminary nature of our code development, some of the results shown may be irnproved in the future. The first result is the comparison of lattice dependence of the halo generation. The results of the halo growth of uniform, FODO, and doublet lattice are shown in Fig. 8 after 1250 turns of storage inside the ring [14]. These results show that there is no halo growth for uniform lattice, less than $10^{-3}$ halo growth for FODO lattice, and there is $1-2 \%$ halo growth for doublet. This may have implication of choosing lattice design for

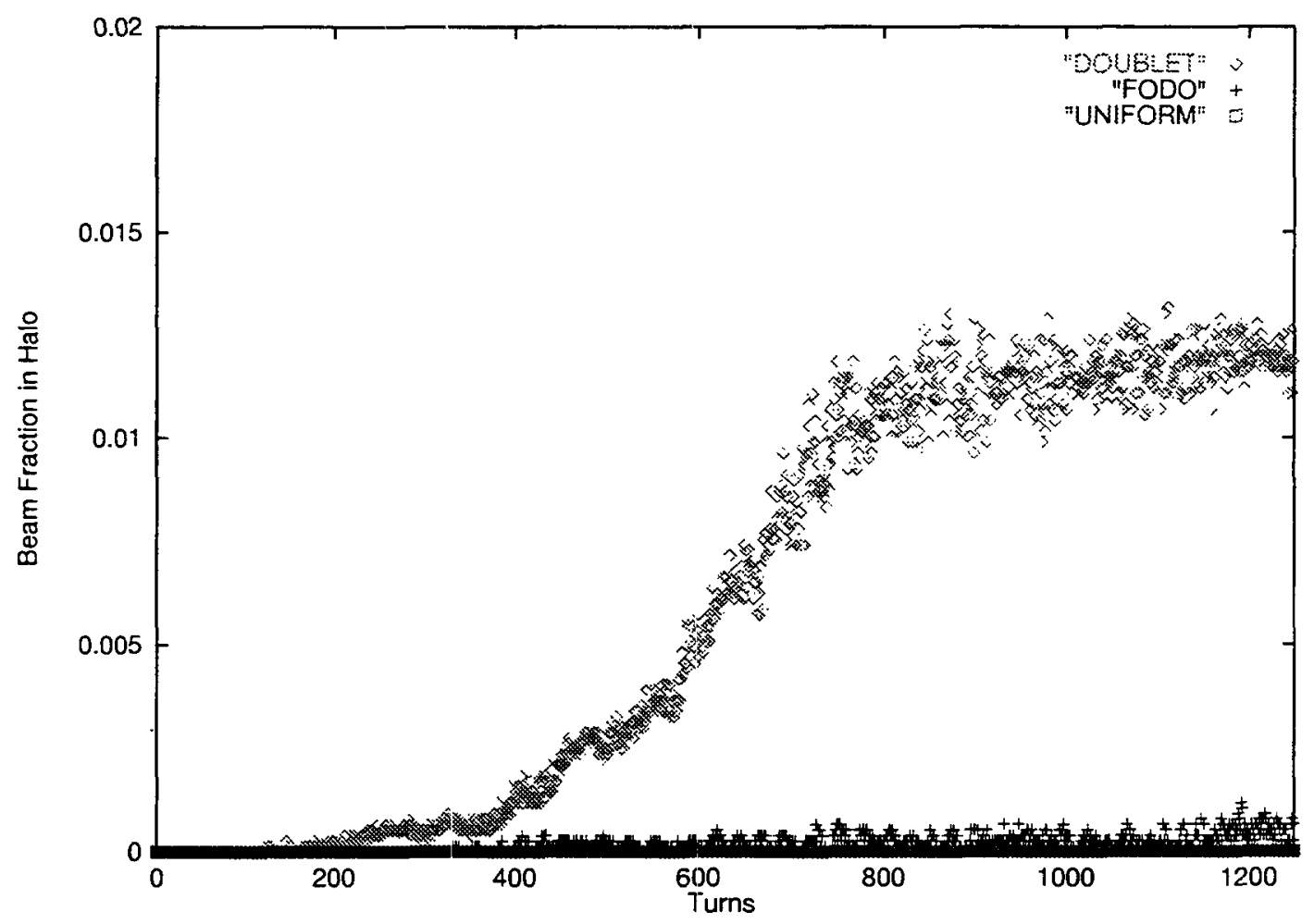

FIGURE 8. Halo growth for uniform, FODO and doublet lattice. 
the accumulator ring to minimize the halo growth during injection.

Applying the PIC and particle-core model to the realistic injection study for the SNS ring, the particle distribution as a function of emittance is plotted in Fig. 9 for one of the injection scenario in vertical plane [13]. It can be seen that the result indicates that there is no particle outside of the $120 \pi$ emittance using old tracking code, but there is a few percent of particles fall outside of $180 \pi$ boundary. We would like to emphasize again that those results obtained here are very preliminary. There is a tendency for a PIC to overestimate the space charge effect if there are not sufficient number of particles in each bin. We will continue this study with more particles and more bins to search for convergence and reliability.

To contain those particles inadvertently migrating toward the wall, after all careful considerations and provisions, a collimator system has to be designed to catch the bulk of them before hitting the wall. For example, for the SNS four collimators, $3.2 \mathrm{~m}$ each, enclosing a $4 \pi$ solid angle around the source point and stuffed with

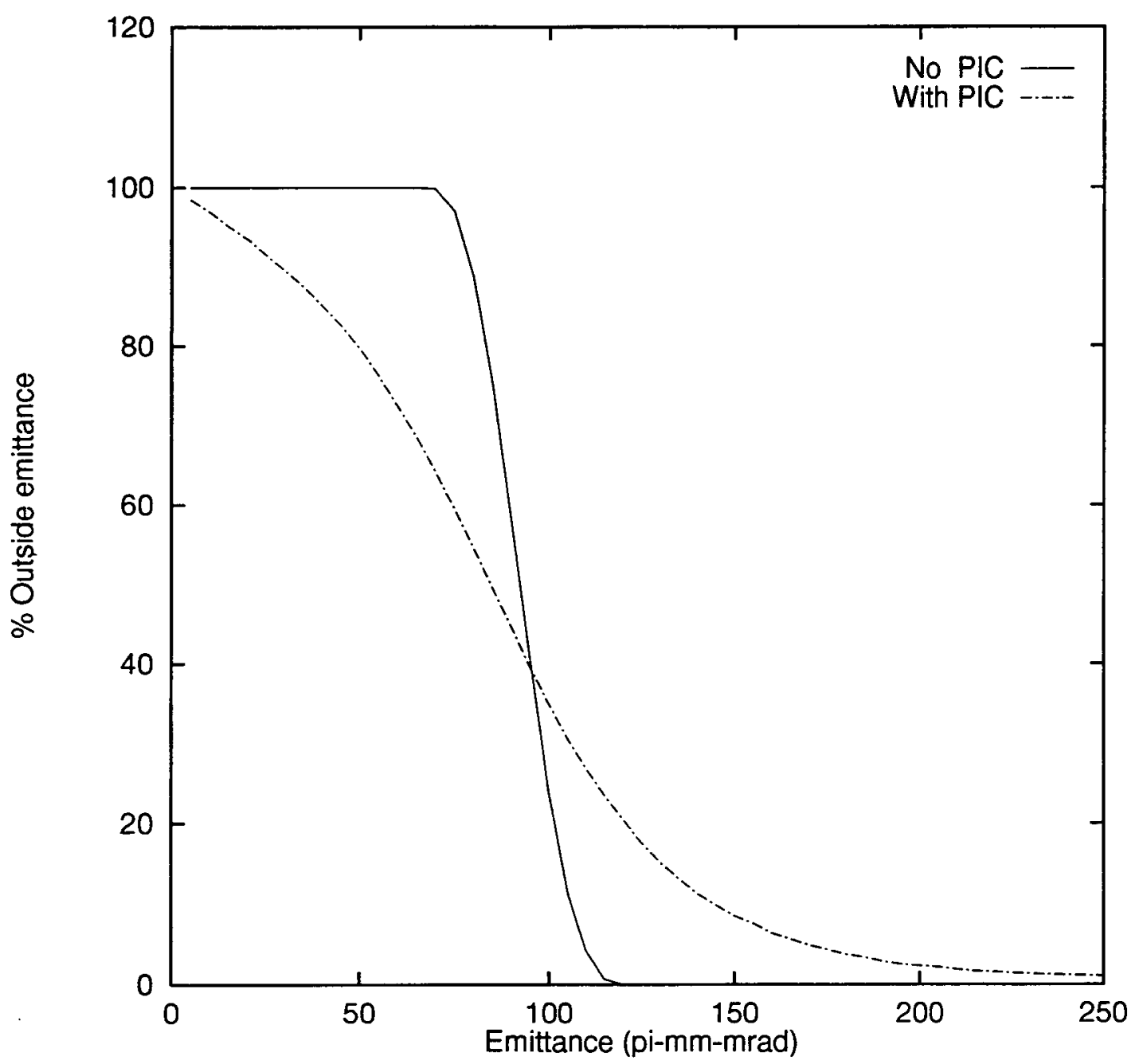

FIGURE 9. Particle distribution calculated by PIC and non-PIC methods. 
segmented material to capture all secondary particles generated by the incident protons will be provided to reduce the radiation effects by a factor of 100 . This way, most of uncontrolled losses will occur at the collimator, leaving ring components relatively intact for reliable operation [15].

For the SNS ring, the beam emittance is $120 \pi$ and the physical acceptance is $360 \pi$. The hard decision is where to put the collimator? The possibility could be around $220 \pi$ to $260 \pi$. The good answer can only be provided by the results from a reliable tracking code incorporating full space charge effects. We will continue to work in this direction to find satisfactory solution for SNS. One way to minimize the particle interception by the collimator is to introduce bend crystal upstream of the collimator to bring large amplitude particles outside of the accumulator ring. Depending on the final strategy adopted, the collimator will take a few percent to a few tenth of a percent beam losses and should be able to sustain direct hit of full beam for few pulses. With the help from this workshop, we will incorporate all the good ideas suggested to improve on the SNS design.

\section{ACKNOWLEGEMENT}

The report given here is the results of both AGS and SNS accelerator physics staff. Contributions by J. Beebe-Wang, M. Brennan, J. Galambos, C. Gardner, J. Holmes, S.Y. Lee, Y.Y. Lee, A. Luccio, and D. Olsen are appreciated. The author would also like to thank J. Beebe-Wang for preparing this manuscript for publication.

\section{REFERENCES}

1. W.T. Weng, et al., "Accumulator Ring Design for the NSNS Project", contributed paper to PAC97, May 12-16, 1997, Vancover, Canada.

2. Y.Y. Lee, C.J. Gardner and A.U. Luccio: "Accelerator Ring Lattice for the National Spallation Neutron Source", contributed paper to PAC97, May 12-16, 1997, Vancover, Canada.

3. L.N. Blumberg and Y.Y. Lee: " $H^{-}$Charge Exchange Injection Into the $1 \mathrm{GeV}$ NSNS Accumulator", BNL/NSNS Tech. Note \#3, November, 1996.

4. A.U. Luccio, J. Beebe-Wang and D. Maletic: "Proton Injection and RF Capture in the National Spallation Neutron Source", contributed paper to PAC97, May 12-16, 1997, Vancover, Canada.

5. W.T. Weng, "Performance and Measurements of the AGS and Booster Beams", AIP Conf. Proc. No.377, p.145, Bloomington, IN, Oct. 10-13, 1995.

6. T. Roser: "High Intensity Performance and Upgrades at the Brookhaven AGS", in these workshop proceedings.

7. L.J. Laslett: "On Intensity Limitations Imposed by Transverse Space-Charge Effects in Circular Particle Accurnulators", BNL Report 7534, p.324-67, 1963. 
8. R.A. Jameson, "Self-Consistent Beam Halo Studies and Halo Diagnostic Development", Frontiers of Accelerator Technology, World Scientific, p.530, November, 1994.

9. S.Y. Lee and A. Riabko, "Envelop Hamiltonian of an Intense Charged-Particle Beam in Periodic Solenoidal Fields", Phys. Rev. E, Vol.51, Feb., 1995.

10. T. Wangler, "Space Charge in Proton Linacs", in these workshop proceedings.

11. A.U. Luccio, J. Beebe-Wang, M. Blaskiewicz, J. Galambos, J. Holmes and D. Olsen, "A Particle Simulation Code for a High Intensity Accelerator Ring", in these workshop proceedings.

12. H. Schonauer, "Addition of Transverse Space Charge to ACCSIM Code", Triumf Design Note TRI-DN-89-K50.

13. J. Galambos, J. Holmes, D. Olsen, J. Whealton, M. Blaskiewicz, A. Luccio and J. Beebe-Wang, "Progress Towards Understanding Transverse Space Charge Effects on SNS Ring Injection", in these workshop proceedings.

14. J.A. Holmes, J.D. Galambos, J.H. Whealton, D.K. Olsen, M. Blaskiewicz, A. Luccio, J. Beebe-Wang and S.Y. Lee, "Space Charge Calculations in Rings for Uniform, FODO, and Doublet Lattice", in these workshop proceedings.

15. H. Ludewig, S. Mughabghab and M. Todosow, "NSNS Ring System Design Study, Collimation and Shielding", BNL/SNS Tech. Note No.5, 1996. 